\title{
Papers
}

\section{Double blind, randomised study of continuous terbinafine compared with intermittent itraconazole in treatment of toenail onychomycosis}

\author{
E Glyn V Evans, Bárdur Sigurgeirsson for the LION study group
}

\begin{abstract}
Objective To compare the efficacy and safety of continuous terbinafine with intermittent itraconazole in the treatment of toenail onychomycosis.

Design Prospective, randomised, double blind, double dummy, multicentre, parallel group study lasting 72 weeks.

Setting 35 centres in six European countries. Subjects 496 patients aged 18 to 75 years with a clinical and mycological diagnosis of dermatophyte onychomycosis of the toenail.

Interventions Study patients were randomly divided into four parallel groups to receive either terbinafine $250 \mathrm{mg}$ a day for 12 or 16 weeks (groups $\mathrm{T}_{12}$ and $\mathrm{T}_{16}$ ) or itraconazole $400 \mathrm{mg}$ a day for 1 week in every 4 weeks for 12 or 16 weeks (groups $\mathrm{I}_{3}$ and $\mathrm{I}_{4}$ ).

Main outcome measures Assessment of primary efficacy at week 72 was mycological cure, defined as negative results on microscopy and culture of samples from the target toenail.

Results At week 72 the mycological cure rates were $75.7 \%(81 / 107)$ in the $\mathrm{T}_{12}$ group and $80.8 \%(80 / 99)$ in the $\mathrm{T}_{16}$ group compared with $38.3 \%(41 / 107)$ in the $\mathrm{I}_{3}$ group and $49.1 \%(53 / 108)$ in the $\mathrm{I}_{4}$ group. All comparisons $\left(\mathrm{T}_{12} v \mathrm{I}_{3}, \mathrm{~T}_{12} v \mathrm{I}_{4}, \mathrm{~T}_{16} v \mathrm{I}_{3}, \mathrm{~T}_{16} v \mathrm{I}_{4}\right)$ showed significantly higher cure rates in the terbinafine groups (all $\mathrm{P}<0.0001$ ). Also, all secondary clinical outcome measures were significantly in favour of terbinafine at week 72 . There were no differences in the number or type of adverse events recorded in the terbinafine or itraconazole groups.

Conclusion Continuous terbinafine is significantly more effective than intermittent itraconazole in the treatment of patients with toenail onychomycosis.
\end{abstract}

\section{Introduction}

With a prevalence among adults of $2-4 \% 0^{1-4}$ onychomycosis is one of the most common nail diseases and one of the few that are curable, providing it is diagnosed correctly. Systemic treatments for onychomycosis now include terbinafine, an allylamine that is primarily fungicidal, and itraconazole, a triazole that is primarily fungistatic; both represent a major therapeutic advance over griseofulvin in the treatment of this condition. For toenail infections terbinafine is usually taken continuously for 12 weeks, whereas itraconazole is taken either continuously for the same period or intermittently-that is, 1 week in 4 weeks for 12 or 16 weeks.

Two double blind studies which compared the efficacy of continuous treatment with terbinafine and itraconazole in onychomycosis have both shown terbinafine to be significantly superior. ${ }^{5}{ }^{6}$ Because therapeutic concentrations of itraconazole are believed to persist in the nail for a considerable time after systemic treatment is stopped, intermittent therapy-with higher daily doses to achieve and maintain therapeutic concentrations - might be an effective alternative to continuous treatment. Such intermittent treatment is widely used currently to treat onychomycosis and is claimed to be as effective for this indication as both continuous itraconazole and continuous terbinafine. ${ }^{78}$

Few direct comparisons of continuous terbinafine versus intermittent itraconazole for onychomycosis exist and those that do are relatively small open studies. ${ }^{9}$ We conducted the first large scale, double blind comparison of continuous terbinafine with intermittent itraconazole in the treatment of toenail onychomycosis.

\section{Methods \\ Protocol \\ Study outline-We undertook a prospective, randomised, double blind, double dummy, multicentre, parallel group study over 72 weeks. A total of 38 investigators from 35 centres in six European countries (Finland, Germany, Iceland, Italy, the Netherlands, and the United Kingdom) participated in the study, the objective of which was to compare the efficacy and safety of continuous terbinafine with inter- mittent itraconazole in the treatment of toenail onychomycosis. \\ Inclusion criteria-Participants were men and women aged 18 to 75 years with a clinical diagnosis of onychomycosis of the toenail (distal subungual or total dystrophic) confirmed by positive results on mycologi- cal culture and microscopy (examination in potassium hydroxide $(\mathrm{KOH}))$. Only patients with dermatophyte infections were included, and all were required to have an affected great toenail (target nail) capable of}

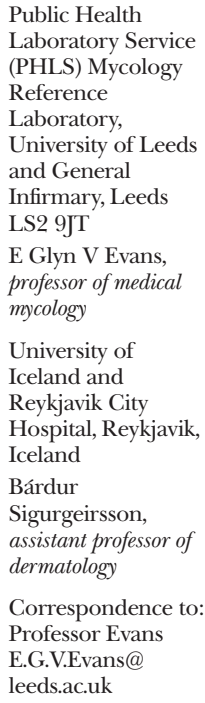

University of Iceland and Reykjavik City Hospital, Reykjavik, Iceland

Bárdur

Sigurgeirsson, assistant professor of dermatology

Correspondence to: Professor Evans E.G.V.Evans@

leeds.ac.uk

BMJ 1999;318:1031-5 
regrowth. Involvement of the great toenail usually occurs in cases that are difficult to treat.

Exclusion criteria-The main exclusion criteria included the use of systemic antifungal treatment in the previous 12 months or topical antifungal treatment within 4 weeks before screening; use of drugs known or believed to interact with either of the study agents; people with conditions that might result in altered absorption, metabolism, or excretion of the study drugs; or radiotherapy, chemotherapy, or immunosuppressive therapy within 12 weeks before the beginning of the study. Other exclusion criteria were pregnancy or lactation; no use of contraception in women of childbearing age; psoriasis, mucocutaneous candidiasis, or immunodeficiency; and alanine transaminase or aspartate transaminase concentrations, or both, more than 1.5 times above the upper limit of the normal range or serum creatinine concentration above $300 \mu \mathrm{mol} / \mathrm{l}$.

Planned interventions-Patients were randomly allocated to one of four groups: terbinafine $250 \mathrm{mg}$ a day for 12 weeks $\left(T_{12}\right)$ or 16 weeks $\left(T_{16}\right)$ or itraconazole $400 \mathrm{mg}$ (four capsules of $100 \mathrm{mg}$ ) a day taken for 1 week in every 4 weeks for either 12 weeks $\left(I_{3}\right)$ or 16 weeks $\left(\mathrm{I}_{4}\right)$. The double dummy system used to blind the study treatments involved the use of placebo tablets and placebo capsules to ensure that all patients took one tablet a day for 16 weeks and four capsules a day for weeks $1,5,9$, and 13 . To facilitate absorption of itraconazole all study medication was taken with meals. Patients were assessed at weeks 4, 8, 12 (end of active treatment for groups $\mathrm{T}_{12}$ and $\mathrm{I}_{3}$ ) and 16 (end of active treatment for groups $T_{16}$ and $I_{4}$ ) and then followed up with blinded assessments at weeks 24, 36, 48, and 72 . Patients were regarded as non-compliant if they failed to take more than two complete daily doses of study medication in any 1 week or more than seven doses in any 1 month; these patients did not continue in the study.

Mycology-All mycological examinations were undertaken at a single laboratory (Public Health Laboratory Service (PHLS) Mycology Reference Laboratory, Leeds).

Ethics-The study protocol conformed to good clinical practice for trials on medicinal products in the European Community; the United States code of federal regulations dealing with clinical studies; and the Declaration of Helsinki on medical research in humans. All patients gave written informed consent to participation in the study and the study protocol was subject to approval by the institutional review board at each study centre.

Primary and secondary outcome measures-The primary efficacy parameter was mycological cure, defined as negative results on microscopy and negative results on fungal culture of samples taken from the target toenail. Rates of mycological cure were assessed at week 72. Secondary efficacy criteria included clinical cure (100\% toenail clearing), complete cure (mycological and clinical cure), clinical effectiveness (mycological cure and at least $5 \mathrm{~mm}$ of new clear toenail growth), and global assessments by physician and patient. Global assessments were performed from weeks 12 to 72 , with both physician and patient basing their assessments on the perceived condition of all affected toenails. The scale used included ratings of very good or excellent (none or minimal signs and symptoms); good (considerable improvement); fair (slight improvement); and poor (no change or worse).

Rationale and methods for statistical analysis-The study was designed to include at least 480 patients (120 in each group). Based on the assumption of a $70 \%$ mycological cure rate with itraconazole the study had $80 \%$ power to detect a $15 \%$ difference at the $5 \%$ level of significance. Treatment comparisons for cure rates (mycological, clinical, complete, and clinical effectiveness) and global assessments were analysed by the Cochran-Mantel-Haenzel test adjusted for country. Results for all efficacy assessments were based on the observed patients of the intention to treat population. Analyses were also performed with the "last observation carried forward" method.

\section{Assignment}

Treatment was assigned according to a predetermined computer generated randomisation code produced by Novartis. Thus study treatments (groups $\mathrm{T}_{12}, \mathrm{~T}_{16}, \mathrm{I}_{3}, \mathrm{I}_{4}$ ) were randomly allotted to sequential patient numbers in balanced blocks of four by centre and investigators then allocated patient numbers sequentially at baseline.

\section{Blinding}

Details of blinding are summarised in table 1. All patients took one tablet a day for weeks 1 to 16 , either active (terbinafine $250 \mathrm{mg}$ ) or placebo. All patients also took two capsules twice daily (total of four capsules daily), either active (itraconazole $100 \mathrm{mg}$ ) or placebo, for weeks 1, 5, 9, and 13. All placebo tablets and capsules were identical in taste and appearance to the respective active compounds. The randomisation code was stored with Novartis and nobody involved in the conduct of the study had access to the code until the database was complete and locked.

\section{Results}

\section{Participant flow and follow up}

A total of 843 patients were screened (figure 1) but 336 of these were excluded because of negative results on fungal culture, withdrawal of consent, protocol violations, or failure to return to the clinic. The 507 remaining patients were randomised for treatment, of whom 506 constituted the study population and 496 the intention to treat population (the 10 patients excluded

Table 1 Details of treatment allocation and blinding. All tablets or capsules given daily

\begin{tabular}{lc}
\hline Treatment group and weeks & Tablets/capsules received \\
\hline$T_{12}$ & 1 terbinafine $(250 \mathrm{mg})$ \\
\hline $1-12$ & 1 placebo tablet \\
\hline $13-16$ & 4 placebo capsules \\
\hline $1,5,9,13$ & 1 terbinafine $(250 \mathrm{mg})$ \\
\hline$T_{16}$ & 4 placebo capsules \\
\hline $1-16$ & 1 placebo tablet \\
\hline $1,5,9,13$ & 4 itraconazole $(100 \mathrm{mg})$ \\
\hline $1-16$ & 4 placebo capsules \\
\hline $1,5,9$ & 1 placebo tablet \\
\hline$I_{4}$ & 4 itraconazole $(100 \mathrm{mg})$ \\
\hline $1-16$ &
\end{tabular}




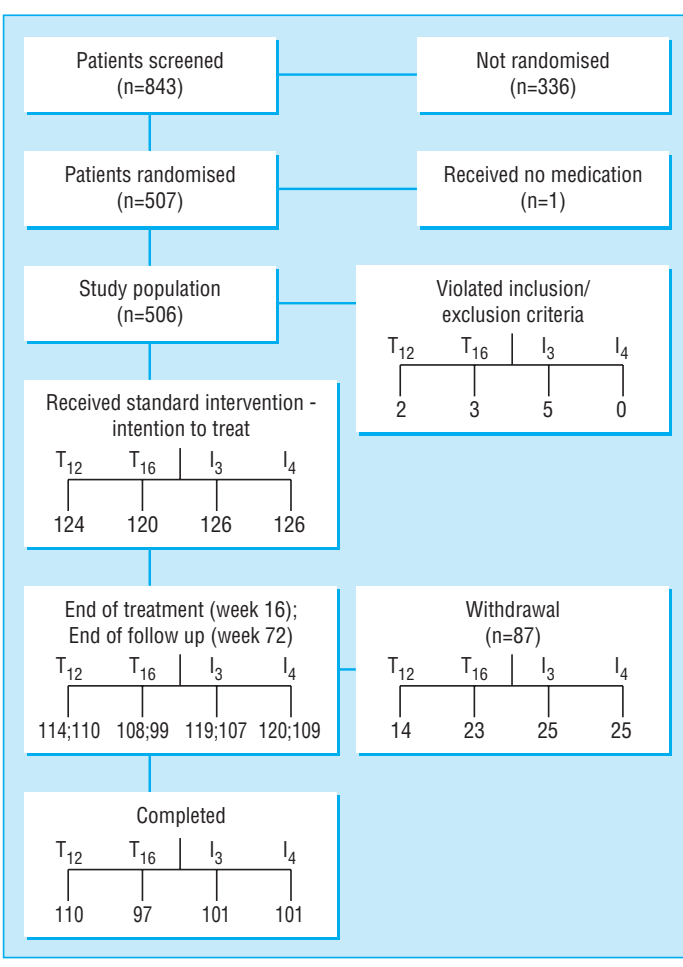

Fig 1 Distribution of participants in study, showing randomisation of intention to treat population between four treatment groups and number of patients remaining in each group at weeks 48 and $72 . \mathrm{T}_{12}$ and $T_{16}$ indicate allocation to terbinafine for 12 or 16 weeks. $I_{3}$ and $I_{4}$ indicate allocation to itraconazole for 1 week a month for 3 or 4 months. Patients not randomised $(\mathrm{n}=336)$ because of negative results on microscopy or fungal culture, or both, at baseline $(n=263)$; protocol violation $(n=19)$; withdrawal of consent $(n=18)$; or other $(n=36)$. Reasons for the 87 withdrawals (for $T_{12}, T_{16}, I_{3}$, and $I_{4}$, respectively) were adverse events $(8,13,5,8)$; withdrawal of consent $(2,1,3,2)$; protocol violation $(0,2,2,5)$; treatment failure $(0,0,8,6)$; failure to return $(4,4,4,4)$; and other $(0,3,3,0)$

violated the inclusion or exclusion criteria but had received at least one dose of medication). There were no significant differences at baseline between the four treatment groups with regard to demographics or to the extent and duration of nail disease (table 2). ${ }^{2}$

\section{Analysis}

Causal agents-The species of dermatophyte isolated at screening were Trichophyton rubrum (443; $89.3 \%)$, T rubrum plus a non-dermatophyte mould (8; $1.6 \%)$, T rubrum plus $T$ mentagrophytes $(3 ; 0.6 \%)$, or $T$ mentagrophytes alone $(42 ; 8.5 \%)$. Also, of all the patients screened for whom a fungus was cultured, dermatophytes were encountered in $95.7 \%(555 / 580)$.

Effects of interventions-Results for all assessments of efficacy for the primary and secondary outcome measures are based on intention to treat. Analyses were also performed with the "last observation carried forward" method. Results did not change with this method nor were there any differences in results when the two populations were analysed separately. Mean compliance was over $99 \%$ in all treatment groups.

\section{Cure rates}

Table 3 and figure 2 summarise the overall cure rates. Differences in mycological cure significantly favoured terbinafine in all comparisons $(\mathrm{P}<0.0001)$, and all comparisons for clinical cure showed significant superiority of both terbinafine regimens versus either of the intermittent itraconazole regimens $(\mathrm{P} \leqslant 0.0022)$. As with the mycological cure rates the clinical cure rates for the continuous terbinafine groups continued to increase after treatment through to week 72 . This was not the case for the intermittent itraconazole groups. All comparisons also showed significantly higher rates of complete cure in the continuous terbinafine groups compared with both the itraconazole regimens $(\mathrm{P} \leqslant 0.0044)$. For clinical effectiveness and global assessments all comparisons showed significantly higher rates of cure for the continuous terbinafine groups $(\mathrm{P}<0.0001)$.

\section{Safety}

A total of 236 patients reported at least one adverse event ( 55 for $\mathrm{T}_{12}, 61$ for $\mathrm{T}_{16}, 60$ for $\mathrm{I}_{3}$, and 60 for $\mathrm{I}_{4}$ ). All were within the known safety profile of both drugs, and there were no significant differences in adverse events between the four treatment regimens. The most commonly reported adverse events were nausea, headache, upper respiratory tract infection, chest infection, back pain, flu-like symptoms, bronchitis, and fever, although most were considered by the investigators to be mild or moderate and unrelated to the study medications.

\section{Discussion}

We have shown in this study that terbinafine $250 \mathrm{mg}$ a day over 12 or 16 weeks produces better mycological and clinical cure rates at week 72 than intermittent itraconazole given over the same periods. Rates of

Table 2 Baseline demographics of patients enrolled in study according to treatment for toenail onychomycosis

\begin{tabular}{|c|c|c|c|c|c|c|}
\hline Parameter & $\begin{array}{l}\text { Terbinafine } 12 \\
\text { weeks }\end{array}$ & $\begin{array}{c}\text { Terbinafine } 16 \\
\text { weeks }\end{array}$ & $\begin{array}{c}\text { Itraconazole } 3 \\
\text { cycles }\end{array}$ & $\begin{array}{c}\text { Itraconazole } 4 \\
\text { cycles }\end{array}$ & All patients & Overall $P$ value \\
\hline No of patients & 124 & 120 & 126 & 126 & 496 & \\
\hline Mean (SD) age (years) & $50.1(12.3)$ & $50.9(13.1)$ & $50.7(12.6)$ & $48.6(13.1)$ & $50.1(12.8)$ & 0.4549 \\
\hline Proportion (\%) of women & 42.7 & 43.3 & 42.9 & 38.9 & 41.9 & 0.8637 \\
\hline Race (\% white; \% Asian/Oriental) & $97.6 ; 2.4$ & $99.2 ; 0.8$ & $99.2 ; 0.8$ & $98.4 ; 1.6$ & $98.6 ; 1.4$ & 0.736 \\
\hline \multicolumn{7}{|l|}{ Dermatophytes (\%): } \\
\hline T rubrum & 91.9 & 85.8 & 88.9 & 90.5 & 89.3 & 0.3549 \\
\hline T rubrum plus non-dermatophyte mould & 1.6 & 2.5 & 1.6 & 0.8 & 1.6 & \\
\hline T rubrum plus $T$ mentagrophytes & & & & 2.4 & 0.6 & \\
\hline$T$ mentagrophytes & 6.5 & 11.7 & 9.5 & 6.3 & 8.5 & \\
\hline Mean (SD) No of infected toenails & $5.6(2.8)$ & $6(2.8)$ & $5.6(2.9)$ & $5.9(2.9)$ & $5.8(2.8)$ & 0.5599 \\
\hline Mean (SD) proportion (\%) of target nail involvement & $73.7(25.5)$ & $69.9(25.3)$ & $69.7(26.2)$ & $68.5(29.00)$ & $70.4(26.6)$ & 0.4455 \\
\hline Mean (SD) duration (years) of current episode & $11.8(10.3)$ & $10.5(9.7)$ & $10.4(8.8)$ & $9.9(9.30)$ & $10.6(9.50)$ & 0.4847 \\
\hline
\end{tabular}


Table 3 Overall cure rates for toenail onychomycosis after 72 weeks according to treatment. Figures are numbers (percentage) of patients

\begin{tabular}{lcccc} 
Detail & $\begin{array}{c}\text { Terbinafine 12 } \\
\text { weeks }\end{array}$ & $\begin{array}{c}\text { Terbinafine 16 } \\
\text { weeks }\end{array}$ & $\begin{array}{c}\text { Itraconazole 3 } \\
\text { cycles }\end{array}$ & $\begin{array}{c}\text { Itraconazole 4 } \\
\text { cycles }\end{array}$ \\
\hline Clinical cure & $59 / 110(54)$ & $59 / 98(60)$ & $34 / 107(32)$ & $35 / 109(32)$ \\
\hline Mycological cure & $81 / 107(76)$ & $80 / 99(81)$ & $41 / 107(38)$ & $53 / 108(49)$ \\
\hline Complete cure & $49 / 107(46)$ & $54 / 98(55)$ & $25 / 107(23)$ & $28 / 108(26)$ \\
\hline Clinical efficacy & $67 / 102(66)$ & $67 / 95(71)$ & $29 / 102(28)$ & $35 / 104(34)$ \\
\hline Global assessment: & & & & \\
\hline Patients & $86 / 109(79)$ & $78 / 99(79)$ & $47 / 107(44)$ & $57 / 109(52)$ \\
\hline Physicians $\dagger$ & $87 / 110(79)$ & $84 / 99(85)$ & $56 / 107(52)$ & $60 / 109(55)$ \\
\hline
\end{tabular}

*Patients who assessed their therapeutic results as good or very good.

†Physicians who rated patients' responses to treatment as good or very good.

clinical cure for terbinafine improved clearly and consistently until the 72 week end point, whereas for itraconazole there was no significant improvement beyond week 48. Both drugs were well tolerated, with the adverse events reported or observed within the established tolerability profile of each drug. ${ }^{10}$ The causal fungi seen in this study were the same as noted in previous studies, ${ }^{11}{ }^{12}$ with dermatophytes accounting for the infection in $96 \%$ of the 580 participants screened for whom a fungus was cultured and, by definition, in all 496 patients randomised to treatment.

One possible explanation for the superior efficacy of terbinafine in this study is provided by the reported differences in fungistatic and fungicidal concentrations of the two drugs. Terbinafine has a primary fungicidal action against dermatophyte fungi with mean minimum inhibitory (MIC) and minimum fungicidal (MFC) concentrations of around $0.004 \mu \mathrm{g} / \mathrm{ml}$, while itracona-

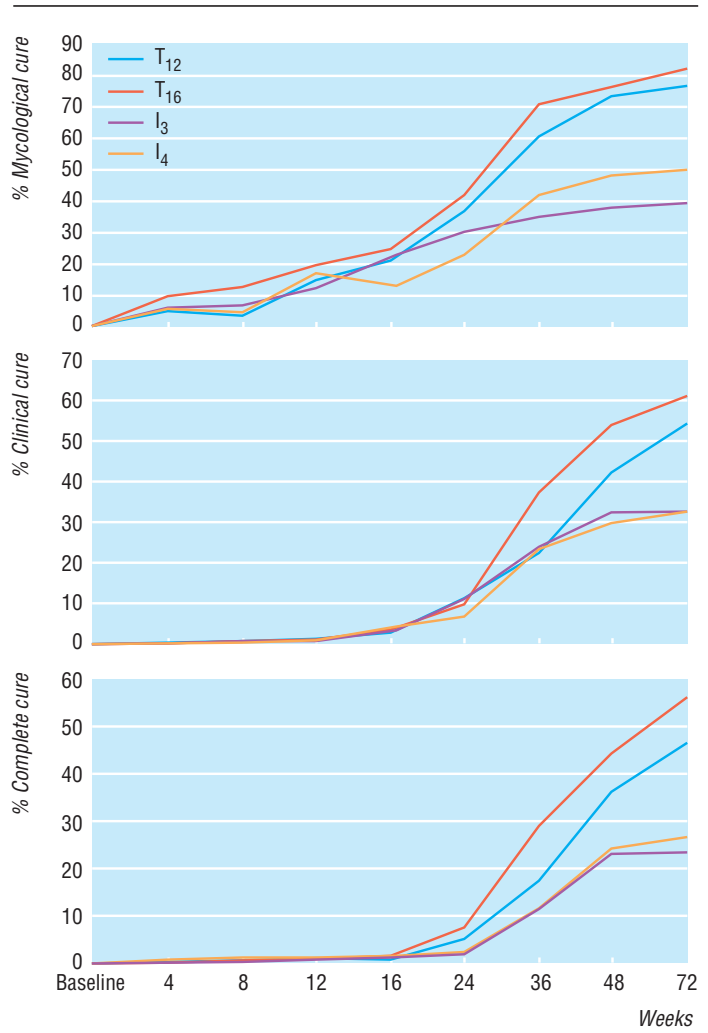

Fig 2 Rates (\%) of mycological cure, clinical cure, and complete cure. All comparisons $\mathrm{P}<0.0001$ except for clinical cure $\mathrm{T}_{12} \mathrm{VI} \mathrm{I}_{3}$ $\mathrm{P}<0.0015, \mathrm{~T}_{12} \vee \mathrm{I}_{4} \mathrm{P}=0.0022$; and for complete cure $\mathrm{T}_{12} \vee \mathrm{I}_{3} \mathrm{P}=0.0007$, and $\mathrm{T}_{12} \vee \mathrm{I}_{4} \mathrm{P}=0.0044$
- Given a correct diagnosis, fungal nail disease (onychomycosis) is curable

- Terbinafine is an allylamine antifungal with a primarily fungicidal mode of action

- Continuous terbinafine treatment over 12 or 16 weeks achieves higher rates of clinical and mycological cure than intermittent itraconazole given over the same periods

- Terbinafine is safe and well tolerated over 12 or 16 weeks of continuous treatment

- Continuous terbinafine should be the current treatment of choice for onychomycosis

zole is primarily fungistatic, with mean MFC of about $0.6 \mu \mathrm{g} / \mathrm{ml}$ in dermatophytes. ${ }^{13}$ Terbinafine concentrations found in the nail are thus around 100-fold higher than the MFC of the drug, ${ }^{14}$ while the reported concentrations of itraconazole are on the borderline between fungistatic and fungicidal action..$^{15}$ If fungicidal action is indeed important for effective clearing of onychomycosis variation in itraconazole concentrations in different patients might influence the therapeutic outcome, while in the case of terbinafine elimination of the pathogen would be achieved despite a wide range of variation. This therapeutic advantage is probably more evident in this study as the participants generally had severe onychomycosis, reflected by a relatively high percentage involvement of the target toenail (mean 70.4\%), longstanding disease (mean 10.6 years), and, on average, onychomycosis in five other toenails.

The principal investigators of the LION study were $\mathrm{J} P$ Steinsson, B Sigurgeirsson, J H Olafsson (Iceland); R Suhonen, T Rantanen, S Stubb, H Heikkilä (Finland); K Gründer, J Ring, M Goos, E G Jung, E Haneke, G Niedergesäss, E Schöpf, P Altmeyer, T Ruzicka, D Reinel (Germany); R E Boelen, L Hamminga, H J van der Rhee, T M Starink, D J Tazelaar, B J Vermeer, J Wuite, D de Hoop, L P Montnor (Netherlands); P Biggio, E di Fonzo (Italy); M J D Goodfield, D T Roberts, J Berth-Jones, D Haworth, I U Haque, C Langdon, V Mittal, R Williams, R Cranfield, R Baldwin (United Kingdom).

We thank Dr Steve Billstein, Novartis Pharmaceuticals Corporation, United States, for his constant help and guidance throughout this project, and Farid Kianifard and Angela Verzilli of Novartis; ICON Clinical Research, Dublin (Ireland), who administered the study and produced the final study report; and the staff of the PHLS Mycology Reference Laboratory, Leeds, who undertook the mycological investigations.

Contributors: EGVE assisted with the planning of the study, was responsible for all the mycological investigations, helped with the interpretation of the results, and was principally responsible for writing and revising the paper. BS was the principal study investigator and as such assisted with the planning of the study and was responsible for the final content of the protocol. BS also made a major contribution to the clinical work of the study, helped with the interpretation of the results, and assisted with writing and revising the paper. Both authors are guarantors.

Funding: Novartis Pharmaceuticals Corporation.

Conflict of interest: EGVE has received funds for research and attending symposia and also fees for speaking and consulting from a number of pharmaceutical companies, including Novartis Pharma and Janssen Pharmaceuticals. BS has received funds for research and fees for speaking and organising educational meetings from several pharmaceutical companies, including Novartis Pharma. Novartis manufactures Lamisil (terbinafine). 
1 Roberts DT. Prevalence of dermatophyte onychomycosis in the United Kingdom: results of an omnibus survey. Br J Dermatol 1992;126(supp 39):23-7.

2 Sais G, Jugglà A, Peyrí J. Prevalence of dermatophyte onychomycosis in Spain: a cross-sectional study. Br J Dermatol 1995;132:758-61.

3 Editorial. Prevalence, morbidity and cost of dermatological diseases. I Invest Dermatol 1979;73:395-401.

4 Heikkilä H, Stubb S. The prevalence of onychomycosis in Finland. $\mathrm{BrJ}$ Dermatol 1995; 133:699-703.

5 de Backer M, de Keyser P, de Vroey C, Lesaffre E. A 12-week treatment for dermatophyte toe onychomycosis: terbinafine $250 \mathrm{mg}$ /day vs itraconazole $200 \mathrm{mg} /$ day - a double blind comparative trial. $\mathrm{Br}$ I Dermatol 1996;134(suppl 136):16-7.

6 Bräutigam M, Nolting S, Schopf RE, Weidinger G for the Seventh Lamisil German Onychomycosis Study Group. Randomised double blind comparison of terbinafine and itraconazole for treatment of toenail tinea infection. BMJ 1995;311:919-21.

Gupta AK, Scher RK. Oral antifungal agents for onychomycosis. Lancet 1998:351:541-2.

8 André J, De Doncker P, Ginter G, Wang R, Stoffels P, Heremans A, et al. Intermittent pulse therapy with itraconazole in onychomycosis: an update [abstract]. 54th Annual Meeting, American Academy of Dermatology, Washington DC, 10-15 February 1996.
9 Tosti A, Piraccini BM, Stinchi C. Treatment of dermatophyte nail infections: an open randomised study comparing intermittent terbinafine therapy with continuous terbinafine therapy and itraconazole therapy. J Am Acad Dermatol 1996;34:595-600.

10 Gupta AK, Sauder DN, Shear NH. Antifungal agents: an overview. Part II. J Am Acad Dermatol 1994:30:911-33.

11 Elewski BE. Large-scale epidemiological study of the causal agents of onychomycosis: mycological findings from the multicenter onychomycosis study of terbinafine. Arch Dermatol 1997;133:1317-8

12 Ellis DH, Marley JE, Watson AB, Williams TG. Significance of non-dermatophyte moulds and yeasts in onychomycosis. Br J Dermato 1997;194(suppl 1):40-2

13 Clayton YM. Relevance of broad-spectrum and fungicidal activity of antifungals in the treatment of dermatomycoses. Br J Dermatol 1994;30(suppl $43) \cdot 7-8$

14 Schatz F, Braütigam M, Dobrowolski E, Effendy I, Haberl H, Mensing H, et al. Nail incorporation kinetics of terbinafine in onychomycosis patients. Clin Exp Dermatol 1995;20:377-83

15 De Doncker P, Decroix J, Piérard GE, Roelant D, Woestenborghs R, Jacqmin P, et al. Antifungal pulse therapy for onychomycosis: a pharmacokinetic and pharmacodynamic investigation of monthly cycles of 1-week pulse therapy with itraconazole. Arch Dermatol 1996;132:34-41. (Accepted 10 February 1999)

\title{
Relation of Chlamydia pneumoniae serology to mortality and incidence of ischaemic heart disease over 13 years in the Caerphilly prospective heart disease study
}

\author{
David P Strachan, David Carrington, Michael A Mendall, Lydia Ballam, Julia Morris, \\ Barbara K Butland, Peter M Sweetnam, Peter C Elwood
}

\begin{abstract}
Objectives To investigate the effect of Chlamydia pneumoniae infection on future development of ischaemic heart disease and mortality.

Design Prospective longitudinal study.

Setting Caerphilly, South Wales.

Subjects Plasma specimens were collected during 1979-83 from 1773 men aged 45-59 years. These were tested for $\operatorname{IgG}$ and $\operatorname{IgA}$ antibodies to $C$ pneumoniae (TW183) by microimmunofluorescence. Outcome measures 13 year mortality and incident ischaemic heart disease events were ascertained from death certificates, hospital records, and electrocardiographic changes at follow up every 4 to 5 years.

Results 642 men (36.2\%) had IgG antibodies at a titre of $\geqslant 1$ in 16 , of whom 362 (20.4\% of all men) also had detectable IgA antibodies. The prevalence of ischaemic heart disease (a history of past or current disease) at entry was similar at all IgG antibody titres but was positively related to IgA antibody titre. IgA antibody titre was positively correlated with plasma viscosity but not with other cardiovascular risk factors. Incidence of ischaemic heart disease was not associated with either IgG antibody titre or IgA antibody titre, but there were stronger and significant relations of IgA antibodies with all cause mortality and fatal ischaemic heart disease, which persisted after adjustment for conventional cardiovascular risk factors. The odds ratios associated with detectable IgA antibodies were 1.07 (95\% confidence interval 0.75 to 1.53) for all incident ischaemic heart disease, 1.83 (1.17 to 2.85 ) for fatal ischaemic heart disease, and 1.50 (1.10 to 2.04 ) for all cause mortality.
\end{abstract}

Conclusion This is the first prospective demonstration of an association between IgA antibodies to $C$ pneumoniae, a putative marker of chronic infection, and subsequent risk of death from ischaemic heart disease. In contrast to earlier case-control studies, IgG antibodies were not associated with either prevalent or incident ischaemic heart disease.

\section{Introduction}

Since the first report of increased concentrations of IgG and IgA antibodies to Chlamydia pneumoniae in patients with acute myocardial infarction or chronic coronary heart disease, ${ }^{1}$ evidence has accumulated of an association between serological markers of this infection and clinically significant atheroma or manifestations of ischaemic heart disease. ${ }^{2}$ The detection, both by polymerase chain reaction or immunocytochemistry ${ }^{3}$ and by culture, ${ }^{4}$ of $C$ pneumoniae in atheromatous plaques lends biological plausibility to a causal link. Although there seems to be preferential localisation of this organism in cardiovascular tissue, ${ }^{5}$ its role in the pathogenesis of atheroma and clinical ischaemic heart disease remains controversial. ${ }^{2}{ }^{6}$ In addition to possible local effects, it has been suggested that persistent $C$ pneumoniae infection may result in altered lipid metabolism, increased fibrinogen concentrations, and low grade systemic inflammation, as shown by increased $\mathrm{C}$ reactive protein concentrations..$^{7-10}$

Most published epidemiological studies have been of cross sectional or case-control design, ${ }^{2}$ in which a spurious association could arise from antigenic cross reactivity between $C$ pneumoniae and damaged cardiac
Department of Public Health Sciences,

St George's

Hospital Medical School, London SW17 0RE

David Strachan, professor

Barbara Butland, lecturer

Department of Medical

Microbiology,

St George's

Hospital Medical

School

David Carrington, senior lecturer

Division of Gastroenterology, Endocrinology and

Metabolism,

St George's

Hospital Medical

School

Michael Mendall,

senior lecturer

Lydia Ballam,

research assistant

Julia Morris,

research assistant

continued over

BMJ 1999;318:1035-40 